\title{
Psychiatry and General Practice Today
}

Edited by lan Pullen, Greg Wilkinson, Alastair Wright \& Denis Pereira Gray

This guide to the assessment and treatment of people with psychiatric disorders in general practice covers clinical syndromes, modern treatment approaches, training, research and prevention. The book places special emphasis on collaboration between general practitioners and psychiatrists and partnership both with patients and their relatives, and between disciplines and agencies.

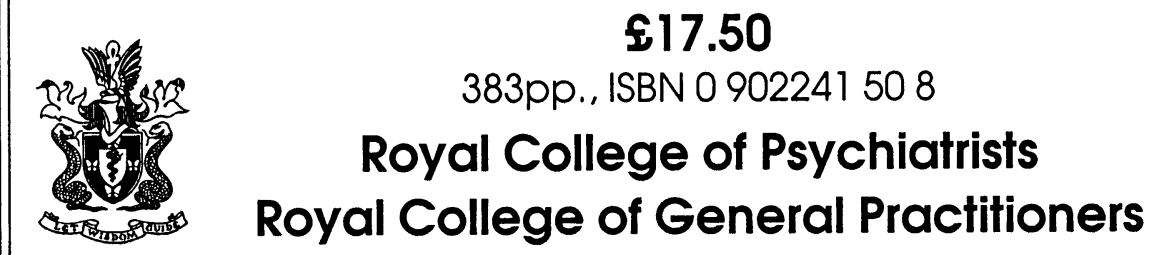

\section{Perinatal Psychiatry}

Use and misuse of the Edinburgh Postnatal Depression Scale

\section{Edited by John Cox \& Jeni Holden}

Research findings and clinical practice are combined in this guide to the screening of women for postnatal disorders, and to the setting up and auditing of services for postnatal depression. All perinatal psychiatric disorders are covered, as well as their effects on infants and partners. Full details are given on the use of the renowned Edinburgh Postnatal Depression Scale, along with translations and instructions for its use in different cultures.

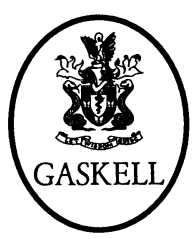

$£ 15.00$ 288pp., ISBN 0902241680

Available from the Publications Department, Royal College of Psychiatrists, 17 Belgrave Square, London SW1X 8PG, tel. 071-235 2351. 
